\title{
Inflammatory aneurysm: A cause of obstructive nephropathy
}

\author{
LARRY D. BUCHANAN, DO \\ WILLIAM HOLDEN, MD
}

\section{Inflammatory aortic}

aneurysms are aneurysms that are surrounded by a variable amount of periaortic fibrosis. This periaortic fibrosis may involve abdominal viscera, such as the duodenum, inferior vena cava, renal veins, or ureters, to a variable degree. On ultrasonographic images, the periaortic fibrosis appears as a hypoechoic mass surrounding the anterior and anterolateral aspects of a dilated aorta. Computed tomography scans reveal a periaortic mass of inflammatory tissue surrounding a dilated atherosclerotic and thickened aorta. The inflammatory mass may enhance to the same extent or slightly less than the aortic lumen following intravenous administration of a contrast agent. Magnetic resonance imaging scans may show the periaortic mass of inflammatory tissue to have a pseudocapsule of compressed fat at its periphery.

Recognition of this entity and the extent of the inflammatory process is important to the definitive management of this condition, as illustrated by the case presented.

In 1935 , James ${ }^{1}$ first described nonmalignant retroperitoneal fibrosis causing obstructive nephropathy. However, only a small number of such cases have been reported in association with abdominal aortic aneurysms, mostly in the surgical ${ }^{2-6}$ or urologic literature, ${ }^{1,7-10}$ with little mention of the subject in the radiologic literature. Radiologists should become familiar with the typical appearance of this disease process on the various imaging modalities of ultrasonography, computed tomography (CT), and magnetic resonance imaging (MRI) to provide accurate preoperative diagnosis and assessment.

The following is a case report of a patient who was seen with an obstructive nephropathy secondary to an inflammatory aortic aneurysm.

\section{Report of case}

A 76-year-old man initially consulted his family physician after two episodes of painless, initial gross hema- turia and left flank pain subsequent to a fall. Because he had no obstructive or other lower urinary tract symptoms, he was treated symptomatically. When he continued to have intermittent left flank pain, he was referred to a urologist for further evaluation.

On initial urologic evaluation, he had the following laboratory values: BUN, $50 \mathrm{mg} / \mathrm{dL}$; creatinine, $4.4 \mathrm{mg}$ / $\mathrm{dL}$; and potassium, $6.3 \mathrm{mmol} / \mathrm{L}$. A bilateral retrograde urogram revealed hydroureteronephrosis and extrinsic compression and medial displacement of the ureters bilaterally at the level of L-4 and L-5 (Figs 1,2).

Attempts at percutaneous nephrostomy and stent placement on the left side were unsuccessful. The patient then was transferred to our institution for further workup and treatment of the obstructive uropathy.

On initial examination at our institution, his past medical history was significant only for a 10 -lb weight loss and anorexia of 2 to 3 months' duration prior to admission. He also had a history of tobacco use exceeding 60 packs per year.

On physical examination, his vital signs were in the normal ranges. A $5.5-\mathrm{cm}$ pulsatile mass was palpated within the midabdomen just superior to the umbilicus. The abdomen was tender to palpation in the left upper and lower quadrants, without evidence of peritoneal signs. Mildly decreased pulses and bruits were detected over the femoral arteries bilaterally. Distal pulses were absent in both lower extremities. The findings of the remainder of the physical examination were essentially normal.

Admission laboratory studies yielded the following values: hematocrit, $21.2 \%$; WBCs, $9800 / \mathrm{cu} \mathrm{mm}$; serum BUN, $50 \mathrm{mg} / \mathrm{dL}$; serum creatinine, $6.2 \mathrm{mg} / \mathrm{dL}$, and serum potassium, $6.8 \mathrm{mmol} / \mathrm{L}$. Urinalysis showed 0 to $2 \mathrm{WBCs}$ per high-power field; RBCs were too numerous to count.

Cystoscopy revealed an adenoma in the lateral lobe of the prostate gland, marked bladder trabeculations, and erythema of the trigone and posterior wall of the bladder. Ureteral stents were passed into the proximal ureters bilaterally. There was hydronephrotic drip of clear urine on the right, while there was a slow drip of bloody urine on the left. With stents in place, the patient's renal function stabilized over the next few days (BUN, $45 \mathrm{mg} / \mathrm{dL}$; creatinine, $6.1 \mathrm{mg} / \mathrm{dL}$ ).

Despite stabilized renal function, the patient complained of persistent left-sided abdominal and flank pain. An abdominal ultrasound examination revealed bilateral hydronephrosis. The abdominal aorta was not well imaged because of overlying bowel gas. Computed tomography scans of the abdomen revealed an abdominal aortic aneurysm measuring $5 \mathrm{~cm}$ in its greatest dimen- 

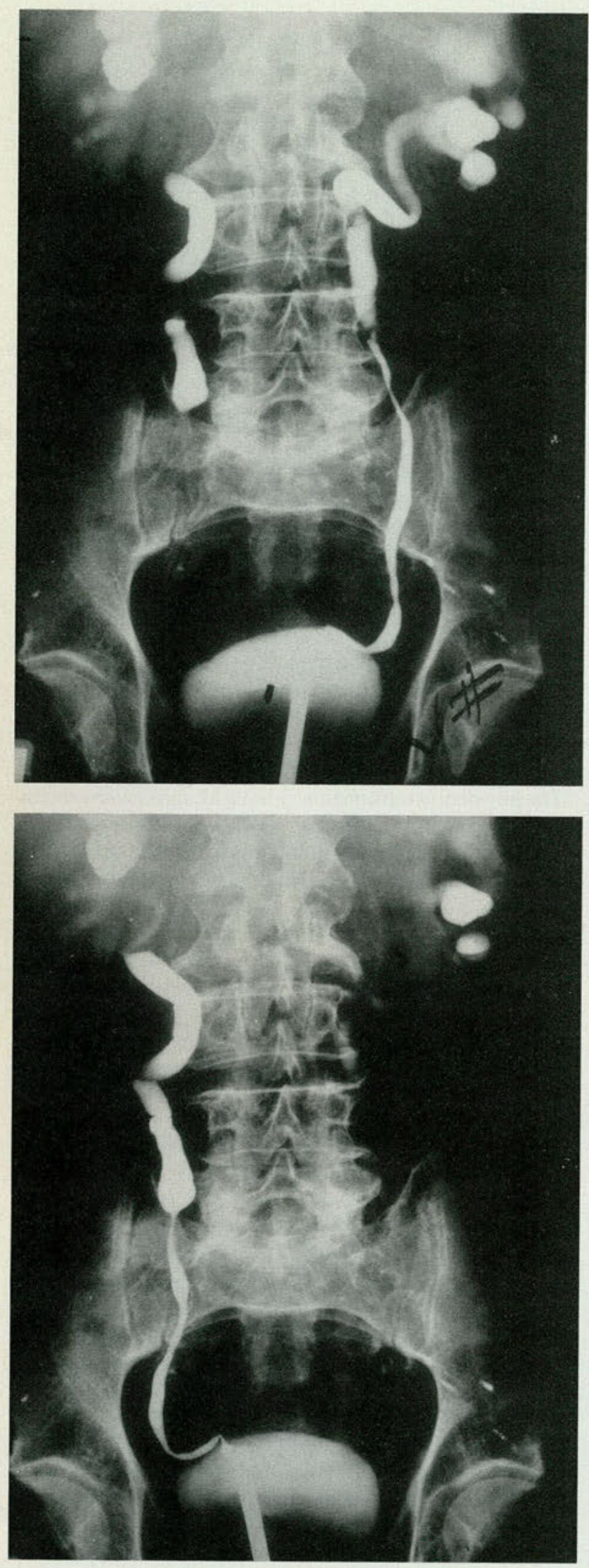

Figure 1. Retrograde urogram on left. Note obstruction of ureter at L-4 level with dilatation proximal to collecting system. There is medial displacement of ureter at level of obstruction.

sion; it extended from just below the level of the renal arteries to the bifurcation of the aorta.

There were dense calcifications in the wall of the abdominal aorta. An ill-defined, soft-tissue density, measuring between 36 and 66 Hounsfield units, surrounded the anterior and lateral aspects of the abdominal aorta and inferior vena cava. This soft-tissue density extended from just below the level of the renal arteries to the level of the common iliac vessels bilaterally.

The ureters coursed within the lateral aspects of this soft-tissue mass at the level of the lower lumbar spine. There was mild medial deviation of the ureters at this level (Fig 3). There also was an irregular, soft-tissue density, measuring approximately 45 Hounsfield units, in the left posterior pararenal space. These periaortic and pararenal soft-tissue densities were thought to represent hemorrhage and possible fibrosis. Because of the patient's elevated serum creatinine level, no intravenous contrast agent was used during these examinations.

On the basis of the CT findings and the patient's persistent abdominal pain and low hematocrit value $(26 \%$ despite transfusion of four units of packed RBCs), surgery was performed for possible leaking abdominal aortic aneurysm. At surgery, the aneurysm was found to be encased in a dense, white, shiny, fibrotic reaction that involved the duodenum, inferior vena cava, common iliac vessels, and ureters at the level of the lower lumbar spine.

There was no evidence of hemorrhage from the aorta. A hematoma, found incidentally in the left posterior pararenal space, was thought to be secondary to prior percutaneous nephrostomy attempts. The abdominal aortic aneurysm was resected, and an aortofemoral bypass was performed using a Dacron graft. Ureterolysis was not attempted at the time of surgery.

The patient's postoperative course was uncomplicated, and he was discharged on the 16th postoperative day. At discharge, the patient's serum BUN level was $42 \mathrm{mg} /$ $\mathrm{dL}$ and serum creatinine, $3.2 \mathrm{mg} / \mathrm{dL}$.

\section{Discussion}

Inflammatory aneurysm of the abdominal aorta, originally thought to be an uncommon variant of abdominal aortic aneurysms, ${ }^{3}$ recently has been recognized as a distinct surgical entity. ${ }^{3}$ It is a disease process that has not been well recognized in the radiologic literature in the past. The reported

Figure 2. Retrograde urogram on right. Note obstruction of ureter at L-5 level with dilatation proximal to collecting system. 

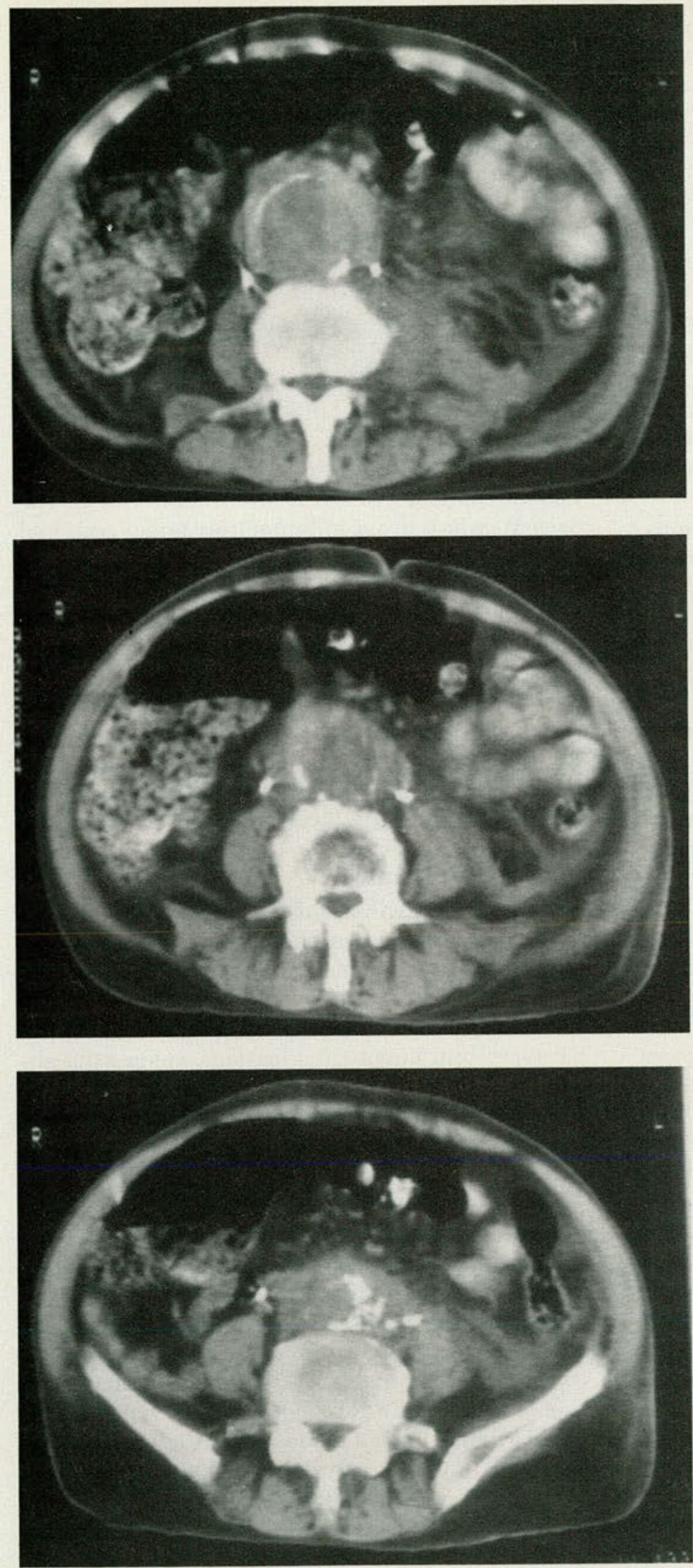

Figure 3. Top: Computed tomography scan of abdomen just inferior to lower pole of kidneys. There is aneurysmal dilatation of aorta with calcification in aortic wall. Perianeurysmal fibrosis surrounds anterior and anterolateral aspects of aorta. Inferior vena cava is compressed at right lateral aspect of aorta. Fibrosis is contiguous with inferior vena cava. Center: Computed tomography scan of abdomen proximal to aortic bifurcation. Perianeurysmal fibrosis involves ureters bilaterally. Inferior vena cava also is involved within perianeurysmal fibrosis and cannot be seen as separate structure at this level. Streaky densities in left retroperitoneum represented a hematoma. Bottom: Computed tomography scan of abdomen at level of aortic bifurcation. Periaortic fibrosis again is seen involving inferior vena cava and ureters at this level. Periaortic fibrosis extended to proximal common iliac vessels bilaterally.

incidence of inflammatory aneurysm has been $5 \%$ to $10 \%$ of all abdominal aortic aneurysms. ${ }^{2,3,11}$ It is characterized by an abnormal perivascular rind of tissue that can range from a subtle obscuration of soft-tissue planes surrounding the aorta and inferior vena cava to a thick periaortic mass of tissue 1.0 to $1.5 \mathrm{~cm}$ in maximal thickness. ${ }^{12,13}$ This perivascular rind or mass of soft tissue frequently involves other retroperitoneal structures, such as the inferior vena cava, renal veins, duodenum, and ureters, to a variable degree.

More than 50 cases of inflammatory aneurysms associated with unilateral or bilateral ureteral obstruction and hydronephrosis have been reported, primarily in the vascular ${ }^{2-5}$ and urologic literature. ${ }^{1,7-10}$ The mechanism of ureteral obstruction appears to be due to ureteral encasement by the periaortic rind of fibrosis. ${ }^{13,14}$

In 1980 , Serra and associates ${ }^{15}$ reviewed 34 reported cases of ureteral obstruction secondary to perianeurysmal fibrosis and found a male-to-female preponderance of 10:1. The average age of the men with this condition was $60.2 \pm$ 1.3 years. The major symptoms in this group of 34 patients were back, flank, or abdominal pain, or a combination of these three. These various locations of pain are most likely secondary to the perianeurysmal reaction in these patients. ${ }^{15}$ Our patient complained of left flank and abdominal pain; however, this pain did 
not begin until after he had fallen onto his left side. Whether this pain was secondary to his retroperitoneal inflammatory process or due to a musculoskeletal injury is uncertain.

\section{Etiology}

The etiology of the perianeurysmal fibrosis has not been established. Originally, Wagenknecht and Madsen ${ }^{7}$ proposed that this perianeurysmal fibrosis was a reaction to microperforations of the aneurysm and subsequent hemorrhage into the retroperitoneal space. This theory, however, seems unlikely because pathologic examinations of tissue samples from various patients have failed to reveal hemosiderin deposits or hemosiderin-laden macrophages as components of this inflammatory reaction.

Bacterial or fungal etiologies of this inflammatory reaction are also unlikely because bacterial and fungal cultures and stains of this inflammatory reaction have been negative. ${ }^{11}$

Labardini and Ratliff ${ }^{8}$ proposed that this fibrosis may represent an exaggeration of the inflammatory response that usually is present in the wall of an aneurysm. In 1986, Cullenward and associates, ${ }^{11}$ proposed an immunologic etiology, with atheromatous plaque material within the aortic wall being a possible allergen source. Whatever the etiology of this fibrosis, the process may be extensive.

\section{Natural history and pathology}

Like the etiology, the natural history of inflammatory aneurysms is poorly understood. Crawford and associates ${ }^{6}$ noted that following repair of an inflammatory aneurysm with an inclusion graft according to the technique advocated by Goldstone and associates, ${ }^{3}$ the periaortic fibrosis tends to regress. They also noted that ureteral patency improved without intervention following regression of the periaortic fibrosis, and they found no signs of fibrosis or recurrent ureteral obstruction in follow-up of these patients. Even though it is uncertain why this inflammatory process subsides following graft insertion, Crawford and associates 6 postulated that the process may be dependent on contact of blood and aortic wall, or alternatively, there may be a natural tendency for the process to subside following surgery.

At gross pathologic examination, inflammatory aneurysms have been found to consist of an outer layer of dense connective tissue, which may include adherent visceral structures, such as the inferior vena cava, renal veins, duodenum, ureters, or sigmoid colon to a variable degree. Microscopically, this outer layer consists of an infiltrate of fibrous tissue, granulation tissue, and a mixed cellular infiltrate consisting primarily of plasma cells, lymphocytes (or aggregates of lymphocytes), neutrophils, and eosinophils. ${ }^{7}$

The underlying aortic wall typically contains fibrocalcific plaques and cholesterol clefts. There is loss of smooth muscle within the media of the aortic wall. The internal elastic lamina shows attenuation and fragmentation. ${ }^{11}$

\section{Laboratory findings}

In his study of 187 patients with abdominal aortic aneurysms (19 had inflammatory aneurysms), Walker and colleagues ${ }^{4}$ found an elevated ESR in approximately $50 \%$ of the patients with inflammatory aneurysms, whereas fewer than $10 \%$ of the patients who had noninflammatory aneurysms had an elevated ESR.

In their patients with inflammatory aneurysms, Goldstone and colleagues ${ }^{3}$ found no clinical or laboratory test that could distinguish this type of aneurysm from noninflammatory aneurysms. Their patients had normal WBC counts (including differential cell counts), negative serologic tests for syphilis, and normal body temperatures. However, the presurgical laboratory evaluation of their patients did not include ESR measurements.

\section{Diagnostic evaluations}

The radiologic evaluation of a patient with an aortic aneurysm who has abdominal, back, or flank pain, or who has symptoms of decreased renal function may begin with a plain film of the abdomen. In $65 \%$ of the cases reported by Culp and Bernatz, ${ }^{9}$ paravertebral linear calcifications, suggesting abdominal aortic aneurysms, were seen on the plain films of the abdomen. Lateral or oblique views of the abdomen also may be helpful in identifying these aortic calcifications.

The urologic evaluation, including intravenous urogram if renal function is not significantly compromised, may reveal varying degrees of unilateral or bilateral hydronephrosis or hydroureter or both down to the level of obstruction. Medial deviation of the involved ureter usually is observed on this examination. This finding is in contrast to that for large noninflammatory aneurysms, which tend to cause lateral deviation of the ureters. ${ }^{10}$

Medial deviation of the ureters, however, is not a specific sign of inflammatory aneurysms; it has been seen in other retroperitoneal processes, such as fibrosis, tuberculosis, or retrocaval ureter. Allibone and Saxton ${ }^{14}$ thought that the association 



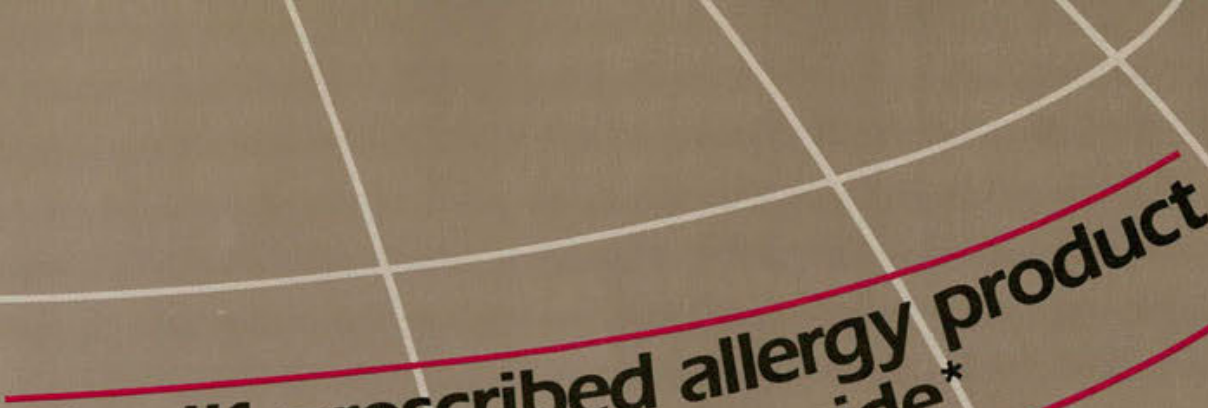

The \#1 prescribed alleride in the U.S. and worldw NO ANTIHISTAMINE - OLD OR NEW IS MORE EFFECTIVE

Maximum efficacy - proven in 30 double-blind clinical studies ${ }^{1-5}$

Fast onset of action - most patients experience significant relief within 1 hour, ${ }^{6+}$ maximum efficacy in 3-4 hours ${ }^{7 *}$

Efficacy maintained with through-the-season use $\mathrm{e}^{8-10}$

Unmatched patient preference- $77 \%$ of patients who started on Seldane and obtained another prescription in the category refilled that prescription with Seldane. (Based upon a 1986 nationwide study of 640 patient members of prepaid group benefit plans.)

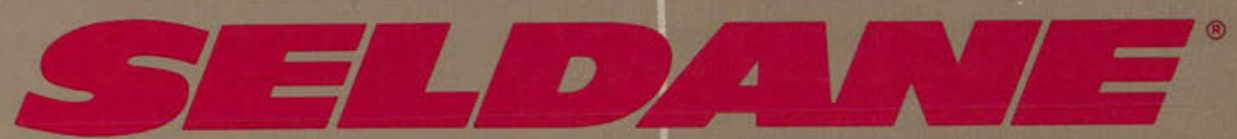

(terfenadine) $60 \mathrm{mg}$ tablets BID

for seasonal allergic rhinitis

The ideal profile for fast, on-the-job relief

* Based upon worldwide prescription and distribution information (1986-1988)-data on file.

** In studies utilizing the histamine-induced skin wheal test. 


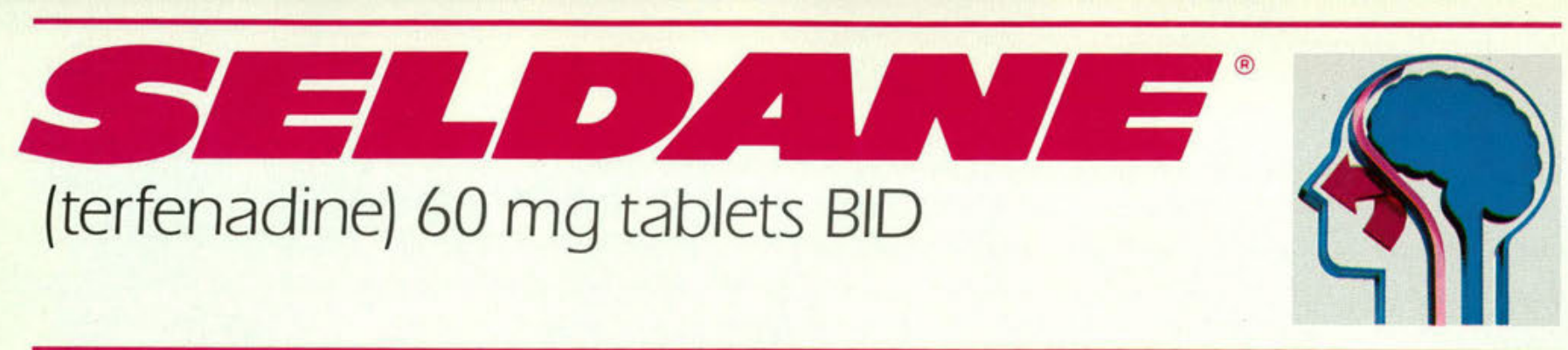

\section{- Fast, unsurpassed relief \\ - Efficacy maintained in through-the-season use \\ B.i.d. dosing for high patient compliance - High patient preference \\ - Lets patients stay alert so they can perform at their best}

\section{Seldane ${ }^{\circledR}$ (terfenadine) $60 \mathrm{mg}$ Tablets \\ BRIEF SUMMARY}

CAUTION: Federal law prohibits dispensing without prescription.

\section{DESCRIPTION}

Seldane (terfenadine) is available as tablets for oral administration. Each tablet contains $60 \mathrm{mg}$ terfenadine. Tablets also contain, as inactive ingredients: corn starch, gelatin, lactose, magnesium stearate, and sodium bicarbonate.

INDICATIONS AND USAGE

Seldane is indicated for the relief of symptoms associated with seasonal allergic rhinitis such as sneezing, rhinorrhea, pruritus, and lacrimation.

\section{CONTRAINDICATIONS}

Seldane is contraindicated in patients with a known hypersensitivity to terfenadine or any of its ingreSeldane
dients.

\section{PRECAUTIONS}

Information for patients

Patients taking Seldane should receive the following information and instructions. Antihistamines are prescribed to reduce allergic symptoms. Patients should be questioned about pregnancy or lactation before starting Seldane therapy, since the drug should be used in pregnancy or lactation only if the potential benefit justifies the potential risk to fetus or baby. Patients should be instructed to take Seldane only as needed and not to exceed the prescribed dose. Patients should also be instructed to store this medication in a tightly closed container in a cool, dry place, away from heat or direct sunlight, and away from children.

Carcinogenesis, mutagenesis, impairment of fertility

Oral doses of terfenadine, corresponding to 63 times the recommended human daily dose, in mice for 18 months or in rats for 24 months, revealed no evidence of tumorigenicity. Microbial and micronucleus test assays with terfenadine have revealed no evidence of mutagenesis.

Reproduction and fertility studies in rats showed no effects on male or female fertility at oral doses of up to 21 times the human daily dose. At 63 times the human daily dose there was a small but significant reduction in implants and at 125 times the human daily dose reduced implants and increased post-implantation losses were observed, which were judged to be secondary to maternal toxicity.

Pregnancy Category C

There was no evidence of animal teratogenicity. Reproduction studies have been performed in rats at doses 63 times and 125 times the human daily dose and have revealed decreased pup weight gain and survival when terfenadine was administered throughout pregnancy and lactation. There are no adequate and well-controlled studies in pregnant women. Seldane should be used during pregnancy only if the potential benefit justifies the potential risk to the fetus.

Nonteratogenic effects

Seldane is not recommended for nursing women. The drug has caused decreased pup weight gain and survival in rats given doses 63 times and 125 times the human daily dose throughout pregnancy and lactation. Effects on pups exposed to Seldane only during lactation are not known, and there are no adequate and well-controlled studies in women during lactation.

Pediatric use

Safety and effectiveness of Seldane in children below the age of 12 years have not been established. General

Consideration should be given to potential anticholinergic (drying) effects in patients with lower airway disease, including asthma.

ADVERSE REACTIONS Experience from clinical studies, including both controlled and uncontrolled studies involving more than
2.400 patients who received Seldane, provides information on adverse experience incidence for periods of a few days up to six months. The usual dose in these studies was $60 \mathrm{mg}$ twice daily, but in a small of a few days up to six months. The usual dose in these studies was $60 \mathrm{mg}$ twice daily, but in
number of patients, the dose was as low as $20 \mathrm{mg}$ twice a day, or as high as $600 \mathrm{mg}$ daily.

In controlled clinical studies using the recommended dose of $60 \mathrm{mg}$ b.i.d., the incidence of reported adverse effects in patients receiving Seldane was similar to that reported in patients receiving placebo. (See Table below.)

ADVERSE EVENTS REPORTED IN CLINICAL TRIALS

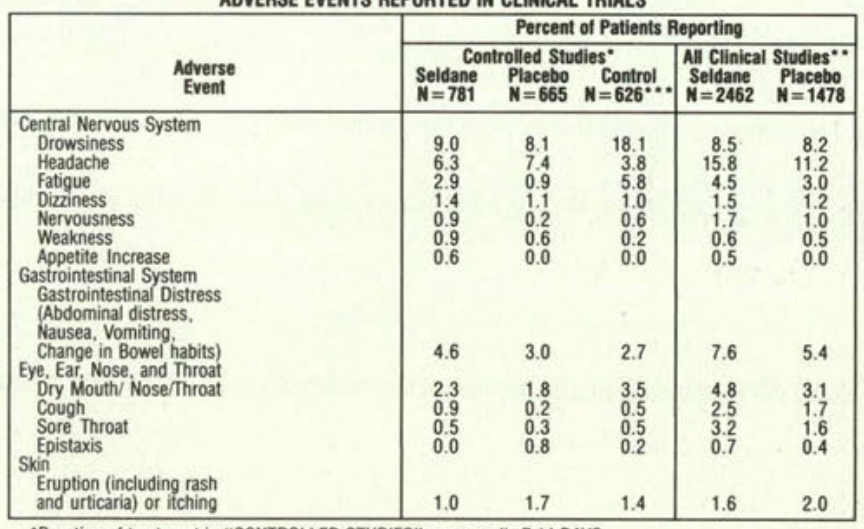

"Duration of treatment in "CONTROLLED STUDIES" was usually 7-14 DAYS

"Duration of treatment in "ALL CLINICAL STUDIES" was up to 6 months.

CONTROL DRUGS: Chlorpheniramine (291 patients), d-Chlorpheniramine (189 patients), Clemastine (146 patients)

In addition to the more frequent side effects reported in clinical trials (See Table), adverse effects have been reported at a lower incidence in clinical trials and/or spontaneously during marketing of Seldane that warrant listing as possibly associated with drug administration. These include: alopecia, (hair loss or thinning), anaphylaxis, angioedema, arrhythmia (including ventricular tachyarrhythmia), bronchospasm, confusion, depression, galactorrhea, hypotension, insomnia, menstrual disorders (including dysmenorrinea), musculoskeletal symptoms, nightmares, palpitation, paresthesia, photosensitivity, proysmention disted in elevations were seen in patients receiving Seldane. Mild elevations were also seen in placebo treated patients. Marketing in patients receiving Selded patients. Marketing experiences include isolated reports of jaundice, cholestatic hepatitis, and hepatitis: mast cas relationship of liver abn is incompleties to Seldane use clear. OVERDOSAGE

Information concerning possible overdosage and its treatment appears in Full Prescribing Information. DOSAGE AND ADMINISTRATION

The usual dosage for adults and children 12 years and older is $60 \mathrm{mg}$ ( 1 tablet) twice daily.

Product Information as of June, 1988

MERRELL DOW PHARMACEUTICALS INC

Subsidiary of The Dow Chemical Company

Cincinnati, Ohio 45215, U.S.A.

Merrell Dow
Y352D

PRINTED IN U.S.A.

References: 1. Kemp JP, Buckley CE, Gershwin ME, et al: Multicenter, double-blind, placebo-controlled trial of terfenadine in seasonal allergic rhinitis and conjunctivitis. Ann Allergy 1985:54:502-509. 2. Backhouse Cl, Brewster BS, Lockhart JDF, et al: Terfenadine in allergic rhinitis. A comparative trial of a new antihistamine versus chlorpheniramine and placebo. Practitioner 1982:226:347-348, 351. 3. Buckley CE. Buchman E. Falliers CJ, et al: Terfenadine treatment of fall hay fever. Ann Allergy 1988;60:123-128. 4. Melillo G. D'Amato G, Zanussi C. et al: A multicentre controlled trial of terfenadine, dexchlorpheniramine, and placebo in allergic rhinitis. Arzneim-Forsch/Drug Res 1982:32:1202-1203. 5. Data on file, MERRELL DOW PHARMACEUTICALS INC.. Cincinnati, Ohio 45215. 6. MurphyO'Connor JC. Renton RL. Westlake DM: Comparative trial of two dose regimens of terfenadine in patients with hay fever. Jint Med Res 1984:12:333-337. 7. Hüther KJ, Renftle G. Barraud N. et al: Inhibitory activity of terfenadine in histamine-induced skin wheals in man. Eur J Clin Pharmacol 1977:12:195-199. 8. Kemp JP, Falliers CJ, Fox RW, et al: A multicenter, open study of the non-sedating antihistamine, terfenadine (Seldane\%), in the maintenance therapy of seasonal allergic rhinitis. Ann Allergy 1988:60:349-354. 9. Krause LB. Shuster S: The effect of terfenadine on dermographic wealing. Br J Dermatol 1984:10:73-79. 10. Simons FR, Watson WT. Simons KJ: Lack of subsensitivity to terfenadine during long-term treatment. J Allergy Clin Immunol 1988;82:1068-1075. 

of curvilinear vascular calcification and ureteral narrowing or medial deviation of the ureters, seen in six of eight of their patients with inflammatory aneurysms, should be suggestive of an inflammatory aneurysm.

Once an aneurysm is suspected, further evaluation may include ultrasonography, which is a reliable means of detecting abdominal aortic aneurysms. ${ }^{14}$ The typical appearance of an inflammatory aneurysm on an ultrasonographic image is that of an echogenic and thickened aortic wall, containing calcific foci, surrounded by a hypoechoic mass. This hypoechoic mass, representing the periaortic fibrosis, is seen at the anterior and anterolateral aspects of the aorta. ${ }^{5,11,14}$ Allibone and Saxton ${ }^{14}$ have advocated evaluation of the kidneys for hydronephrosis if an aneurysm is detected by ultrasound examination. As demonstrated by our case, ultrasonography may not adequately evaluate the abdominal aorta due to overlying bowel gas and mesenteric fat.

Computed tomography, another imaging modality for evaluation of abdominal aneurysms and the retroperitoneum, enables consistent visualization of the aorta and accurate determination of its dimensions. Normally, the wall of the aorta is not readily distinguished from intraluminal blood unless calcification is present in the wall.

Contrast material administered intravenously can help to distinguish aortic lumen and intraluminal defects, such as thrombi. The adventitial surface of the abdominal aorta should be smooth without any overlying soft-tissue density or masses. The margins should be distinct from the surrounding retroperitoneal structures, such as the inferior vena cava, psoas muscle, and vertebral bodies.

Computed tomography scans of an inflammatory aneurysm reveal a thickened and calcified aortic wall surrounded anteriorly and anterolaterally by a soft-tissue mass of low attenuation. The posterior aspect of the aorta is spared from this process. An intravenously administered contrast agent enhances the periaortic fibrosis less than or to the same extent as the aortic lumen. This enhancement is thought to be secondary to the enhancing granulation tissue within the perivascular rind of soft tissue. ${ }^{13,16}$ Computed tomography also may accurately define the extent of the inflammatory aneurysm and the periaortic fibrosis as well as evaluate any involvement of the inferior vena cava, renal veins, duodenum, ureters, or sigmoid colon.

Computed tomography also enables evaluation of the ureters along their course, as well as accurate detection of the level of a ureteral obstruc- tion. Contiguous slice imaging in the region of a ureteral narrowing or stenosis facilitates evaluation of the etiology of the obstruction. In our patient, the ureters were thought to be obstructed and medially deviated due to involvement within the perianeurysmal soft tissue rind in the region of the lower lumbar spine.

Aortography has been useful for defining the size of the aortic lumen, which may be narrowed or dilated, and for defining the extent of the atherosclerotic changes of the aorta. Aortography, however, provides little information regarding the size or extent of the periaortic fibrosis.

Within the past few years, MRI has been used to evaluate inflammatory aneurysms. ${ }^{17}$ The aortic lumen and periaortic inflammatory rind have been well delineated. In some cases, a pseudocapsule of compressed fat has been seen accentuating the periphery of the periaortic inflammatory mass. ${ }^{17}$ The magnetic resonance images may be degraded due to motion artifact from respiration or aortic pulsation. Confusion also has occurred secondary to the variable signal intensity of the atheromatous changes in the wall of the aorta and turbulent flow in the aortic lumen. ${ }^{11,17}$

The differential diagnosis of a periaortic softtissue mass with various degrees of ureteral involvement should include lymphoma, retroperitoneal fibrosis, metastatic disease, and periaortic hemorrhage. With lymphoma, one generally would not expect enhancement after intravenous administration of a contrast agent. Metastatic tumor infiltration in the retroperitoneum may enhance due to its desmoplastic reaction; however, one would expect metastatic tumor to be more extensive in the retroperitoneum than just in a periaortic location. ${ }^{18}$

In idiopathic retroperitoneal fibrosis, the appearance is not so typically periaortic in location and usually is demonstrated on CT scans as a plaquelike fibrotic mass with a density similar to that of the psoas muscle. ${ }^{19}$ Periaortic hemorrhage also has a predilection for ureteral involvement, and the appearance may be similar to that of perianeurysmal fibrosis. However, the extravasation of blood in the retroperitoneal space appears more streaky and usually is not confined to the immediate periaortic area. ${ }^{20,21}$

\section{Management}

Even though the reported incidence of rupture of inflammatory aneurysms is $15 \%,{ }^{4}$ compared with $40 \%$ for the commonly occurring form of aneurysm, ${ }^{4}$ the current approach to management is sur- 
gical repair with inclusion graft. ${ }^{11,21}$ Ureterolysis is advocated only when severe bilateral obstruction or uremia may be present. ${ }^{21}$

Some authors ${ }^{2,5}$ advocate administering steroid therapy preoperatively to decrease the inflammatory process (thereby reducing visceral involvement and allowing ureteral decompression), thus allowing the possibility of simplifying the surgical procedure. However, the use of steroids remains controversial. The inflammatory mass surrounding the aorta in an inflammatory aneurysm actually may reinforce the dilated aorta. By using steroids to decrease the inflammatory mass, the risk of rupture of the aneurysm may be increased. If the luminal diameter of the aorta increases while the patient is on steroid therapy, acute surgical intervention may be required. In patients who may not be surgical candidates, steroid therapy may be useful to decrease the inflammatory process and improve symptoms. Further investigation is needed regarding the use of steroids in the management of inflammatory aneurysms. ${ }^{11}$

\section{Summary}

Inflammatory aortic aneurysms have been reported to account for approximately $5 \%$ to $10 \%$ of all abdominal aortic aneurysms. ${ }^{3,12}$ Recognition of the typical appearance of an inflammatory aneurysm on ultrasonographic images, CT scans, and magnetic resonance images is important in the diagnosis of this entity. Thorough radiologic evaluation of the extent of the inflammatory process and involvement of adjacent viscera, including the duodenum, inferior vena cava, renal veins, and ureters, is important in preoperative planning to decrease surgical morbidity. Recognition of the radiographic appearance of this entity is important in the evaluation of the effectiveness of steroid therapy in the long-term medical management of patients considered to be nonsurgical candidates. By recognizing the typical radiographic appearance of an inflammatory aneurysm, the diagnosis may be suggested prior to surgical intervention. The chance of confusing this entity with a leaking aortic aneurysm, as occurred in the case presented, may be reduced.

1. James TG: Uraemia due to aneurysm of abdominal aorta. $B r J$ Urol $1935 ; 7: 157$

2. Darke SG, Glass RE, Eadie DGA: Abdominal aortic aneurysm: Perianeurysmal fibrosis and ureteric obstruction and deviation. $\mathrm{Br} J \mathrm{Surg}$ 1951;64:649-651.

3. Goldstone J, Malone JM, Moore WS: Inflammatory aneurysms of the abdominal aorta. Surgery 1978;83:425-430.
4. Walker DI, Bloor K, Williams G, et al: Inflammatory aneurysms of the abdominal aorta. Br J Surg 1972;59:609-614.

5. Henry LG, Doust B, Korns ME, et al: Abdominal aortic aneurysm and retroperitoneal fibrosis: Ultrasonographic diagnosis and treatment. Arch Surg 1978;113:1456-1460.

6. Crawford JL, Stowe CL, Safi HJ, et al: Inflammatory aneurysms of the aorta. J Vasc Surg 1985; 2(1):113-124.

7. Wagenknecht LV, Madsen PO: Bilateral ureteral obstruction secondary to aortic aneurysm. $J$ Urol 1970;103:732-736.

8. Labardini MM, Ratliff RK: The abdominal aortic aneurysm and the ureter. J Urol 1967;98:590-596.

9. Culp OS, Bernatz PE: Urologic aspects of lesions in the abdominal aorta. J Urol 1961;86:189-195.

10. Pahira JJ, Wein AJ, Barker CF, et al: Bilateral complete ureteral obstruction secondary to an abdominal aortic aneurysm with perianeurysmal fibrosis: Diagnosis by computed tomography. J Urol 1979;121:103106.

11. Cullenward MJ, Scanlan KA, Pozniak MA, et al: Inflammatory aortic aneurysm (periaortic fibrosis): Radiologic imaging. Radiology 1986;159:75-82.

12. Aiello MR, Cohen WN: Inflammatory aneurysm of the abdominal aorta. J Comput Assist Tomogr 1980;4:265-267.

13. Feldberg MA, van Waes PF, ten Haken GB: CT diagnosis of perianeurysmal fibrotic reactions in aortoiliac aneurysm. $J$ Comput Assist Tomogr 1982;6:465-471.

14. Allibone GW, Saxton HM: The association of aortoiliac aneurysms with ureteral obstruction. Urol Radiol1979-1980;1:205-210.

15. Serra RM, Engle JE, Jones RE, et al: Perianeurysmal retroperitoneal fibrosis: An unusual cause of renal failure. Am J Med 1980;68:149-153. 16. Megibow AJ, Ambos MA, Bosniak MA: Computed tomographic diagnosis of ureteral obstruction secondary to aneurysmal disease. Urol Radiol 1979-1980;1:211-215.

17. Lee JKT, Ling D, Heiken JP, et al: Magnetic resonance imaging of abdominal aortic aneurysms. AJR 1984;143:1197-1202.

18. Ambos MA, Bosniak MA, Megibow AJ, et al: Ureteral involvement by metastatic disease. Urol Radiol 1979;1:105-112.

19. Fagan CJ, Larrieu, AJ, Amparo EG: Retroperitoneal fibrosis: Ultrasound and CT features. AJR 1979;133:239-243.

20. Korobkin M, Callen PW, Fisch AE: Computed tomography of the pelvis and retroperitoneum. Radiol Clin North Am 1979;17:301-319.

21. Sagel SS, Siegel MJ, Stanley RJ, et al: Detection of retroperitoneal hematoma by computed tomography. AJR 1977;129:403-407.

The views expressed in this article are those of the authors and do not reflect the official policy or position of the Department of the Navy, Department of Defense, or the United States Government.

From the department of radiology, Naval Hospital, San Diego.

Reprint requests to Dr Buchanan, department of radiology, Metropolitan Hospital, 1919 Boston SE, Grand Rapids, MI 49506. 


\section{Department of Family Medicine Clinician-Emergency Medicine Ohio University - College of Osteopathic Medicine Athens, Ohio}

The Ohio University College of Osteopathic Medicine is seeking a physician (M.D. or D.O.) for a full-time, tenure track, clinical faculty position in family medicine. The successful candidate will have special interest and qualifications in the practice and teaching of emergency medicine. The position is in the Department of Family Medicine, Section of Emergency Medicine.

Qualifications: Prefer Board-certified and/or residency-trained individual

\section{Responsibilities:}

1. Fulfill clinical duties as assigned in the Emergency Department at O'Bleness Memorial Hsopital.

2. Teach coursework assigned to the Department of Family Medicine with priority given to the teaching of emergency medicine sequences.

3. Maintain, demonstrate, and teach the clinical application of emergency medicine in ambulatory and hospital-based practice.

4. Participate in the ongoing programs of the Department of Family Medicine, including the training of fellows, interns, and residents; and research, as appropriate.

Rank: Assistant/Associate Professor (commensurate with experience/credentials)

Salary: Base salary - Assistant Professor: $\$ 28,999-\$ 36,000$

Associate Professor: $\$ 36,000-\$ 42,000$

\section{PLUS}

Practice Plan salary - negotiated according to clinical capabilities

and faculty service

Research facilities available.

Rural environment with cultural benefits of university town.

Excellent fringe benefits.

Closing Date: September 1, 1989

Starting Date: September 15, 1989

Ohio University is an Affirmative Action/Equal Opportunity Employer

Application may be made to: Anthony G. Chila, D.O., Professor and Chairman

Department of Family Medicine

Grosvenor Hall

Ohio University - College of Osteopathic Medicine

Athens, Ohio 45701

PH: $614 / 593-2210$

$800 / 345-1440$, ext. 2210 (Ohio)

$800 / 345-1560$, ext. 2210 (out-of-state) 